\title{
Panorama de publicações internacionais sobre autoeficácia
}

\section{Overview of international publications on self efficacy}

\author{
CRISLEI DAIANA OLIVEIRA SIQUEIRA SCHUCHE \\ DEBORA BOBSIN ID \\ SIMONE ALVES PACHECO DE CAMPOS (D) \\ GLAUCO DE OLIVEIRA RODRIGUES D
}

\section{RESUMO}

O presente estudo tem como objetivo analisar o panorama geral das as publicações sobre autoeficácia, na área de gestão, a partir de uma revisão bibliométrica. A revisão abrange a pesquisa empírica sobre autoeficácia publicada entre os anos 2000 até 2020, na base de dados Web of Science (WOS). O estudo identificou e compilou as principais informações referentes às publicações contendo a palavra-chave self efficacy no título, na categoria de Gestão e Negócios, perfazendo um total de 519 artigos incluídos na revisão. Por meio de uma abordagem qualitativa e quantitativa, buscou-se qualificar e quantificar determinadas variáveis relacionadas à literatura específica referente ao tema. Os estudos sobre o tema demonstram como pessoas autoeficazes são melhores funcionários no sentido que desempenham melhor suas funções. Os principais resultados da pesquisa mostram que os estudos relacionados ao tema têm se diversificado nos últimos vinte anos, embora sobrepõe-se estudos envolvendo a autoeficácia empreendedora, a criativa e a ocupacional. Além disso, observou-se um maior número de publicações em 2019, resultado que influencia no incremento de pesquisas desta última década, atingindo um acréscimo de $250 \%$, se comparado com a década 
anterior. Finalmente, deve-se enfatizar que as publicações sobre autoeficácia buscam investigar o desempenho dos funcionários e identificar os fatores que o influenciam, uma das prioridades mais importantes no gerenciamento de recursos humanos.

Palavras chave: autoeficácia, gestão de recursos humanos e bibliometria

\section{Abstract}

This study aims to analyse the general panorama of publications on self-efficacy, in the area of management, based on a bibliometric review. The review covers empirical research on self-efficacy published in the period between 2000 and 2020, in the Web of Science (WOS) database, in peer-reviewed academic journals. This study identified and compiled the main information related to publications of the documentary type article, containing the keyword self efficacy in the title, in the Management and Business category, totalling 519 articles included in the review. Through a qualitative and quantitative approach, we sought to qualify and quantify certain variables related to the specific literature on the subject. Studies on the subject demonstrate how self-efficacious people are better employees in the sense that they perform their functions better, as they are more efficient, like challenges, know how to act in pressure situations, are more creative and/or entrepreneurial, as the environment requires, since adapt to different situations, among other different characteristics that make them different. The main results of the research show that studies related to the theme have diversified in the last twenty years, although studies involving entrepreneurial, creative and occupational self-efficacy overlap. In addition, there was a greater number of publications in 2019, a result that influences the increase in research in the last decade, reaching an increase of $250 \%$, compared to the previous decade. Finally, it should be emphasized that publications on self-efficacy seek to investigate employee performance and identify factors influencing it, one of the most important priorities in human resource management. Keywords: self-efficacy, human resource management and bibliometric 


\section{INTRODUÇÃo}

A visão da natureza humana incorporada em teorias psicológicas, para explicar o comportamento e os processos causais que elas postulam, têm uma importância considerável (BANDURA, 1999). Para Bandura (1999), à medida que o conhecimento obtido por meio da investigação é aplicado, as concepções que orientam as práticas sociais têm implicações ainda mais vastas. Elas afetam quais potencialidades humanas são cultivadas, quais são subdesenvolvidas e se os esforços de mudança são direcionados principalmente a fatores psicossociais, biológicos ou socioestruturais.

As pesquisas sobre Autoeficácia procuram responder quais seriam os potenciais influenciadores, dos comportamentos dos funcionários, visando atingir os objetivos e resultados das Instituições. Isso porque as crenças de autoeficácia desempenham um papel central no funcionamento humano. Elas influenciam no estabelecimento das metas dos indivíduos e por quanto tempo eles persistem diante de dificuldades e fracassos (BANDURA, 1977a).

Segundo Bandura (1977a,1978), a autoeficácia reflete a crença momentânea de um indivíduo em sua capacidade de realizar uma tarefa específica em um nível específico de desempenho. Wood e Bandura (1989, p. 408) seguem essa mesma linha, definindo a autoeficácia, como as "crenças nas capacidades de alguém para mobilizar a motivação, recursos cognitivos e cursos de ação necessários para atender a determinadas demandas situacionais".

A autoeficácia está positivamente relacionada à aceitação de uma meta difícil mesmo quando o desempenho passado foi controlado, sugerindo que parte do efeito positivo pode estar relacionado à adoção de metas mais difíceis por indivíduos com alta autoeficácia (VANCOUVER; THOMPSON; WILLIAMS, 2001). No entanto, a partir de outras teorias motivacionais (especialmente teorias de metas), pode-se concluir que, embora a alta autoeficácia possa motivar os indivíduos a adotar metas de alto nível, ela pode reduzir a motivação dentro de um nível de meta (VANCOUVER, et al., 2002).

Com relação à formação de crenças de autoeficácia, Bandura (1977a) propôs várias fontes de informação que nutrem a autoeficácia dos indivíduos em situações com as quais eles não estão familiarizados (por exemplo, experiências indiretas por modelos). 
Grether, Sowislo e Wiese (2017) sinalizam que o foco de Bandura (1997) está nas crenças de autoeficácia específicas para tarefas e situações. Entretanto, embora admita que elas possam generalizar-se para além de uma determinada situação, para ele, essa generalização se restringe a situações e atividades muito semelhantes àquelas em que as crenças de autoeficácia foram originalmente desenvolvidas. Em contraste, outros autores colocam uma forma geral de crenças de autoeficácia em primeiro plano (CHEN; GULLY; EDEN; 2001; SCHERBAUM; COHEN-CHARASH; KERN, 2006; SCHWARZER; JERUSALEM, 1995).

Uma noção fundamental subjacente à concepção de autoeficácia de Bandura é que o conjunto de crenças se desenvolve e se cristaliza com o tempo, mas como os estudos não mostram como isso ocorre, vale a pena examinar o desenvolvimento longitudinal das crenças de autoeficácia (AGARWAL; SAMBAMURTHY; STAIR, 2000).

A presente pesquisa indica que os estudos relacionados ao tema da autoeficácia têm se diversificado nos últimos vinte anos. Sendo assim, buscou-se responder a seguinte problemática: Como o tema de autoeficácia, na área de gestão, tem se desenvolvido nos últimos 20 anos? Para responder a tal questão, foi definido o seguinte objetivo geral: analisar o panorama geral das publicações sobre autoeficácia, na área de gestão, a partir de uma revisão bibliométrica. Para o alcance do objetivo geral, foram definidos os seguintes objetivos específicos: a) seleção das bases de dados; b) seleção das palavras-chave adequada; c) seleção do tipo de documento; d) busca de artigos alinhados ao tema; e) categorização dos artigos da amostra selecionada em: título; autores; títulos das fontes; DOI; anos das publicações; palavras chave; palavras chave plus e resumo.

O presente trabalho está estruturado em cinco seções, além desta introdução. A primeira apresenta o contexto teórico da autoeficácia, bem como os temas de autoeficácia específicas mais estudados; a segunda, procedimentos metodológicos; a terceira seção apresenta a análise e discute-se os resultados. Na sequência, revela-se as considerações finais e as referências utilizadas para embasamento teórico do mesmo. 


\section{Autoeficácia}

Bandura (1977a, 1977b) introduziu pela primeira vez o conceito de autoeficácia e o interesse nela tem sido constante desde a década de 1970. Trata-se de um elemento-chave na teoria de aprendizado social de Bandura (1977b), e refere-se à crença na capacidade de realizar uma tarefa específica. Ela surge da aquisição gradual de habilidades cognitivas, sociais, linguísticas e / ou físicas complexas através da experiência (BANDURA, 1982).

A autoeficácia percebida diz respeito às "crenças das pessoas em suas capacidades de mobilizar a motivação, os recursos cognitivos e os cursos de ação necessários para exercer controle sobre os eventos em suas vidas" (WOOD; BANDURA, 1989, p. 364). Para Zhao, Seibert e Hills (2005), autoeficácia é um construto motivacional que demonstrou influenciar a escolha de atividades de um indivíduo, níveis de metas, persistência e desempenho em uma variedade de contextos.

Destaca-se a partir disso a Teoria Social Cognitiva (TSG), tendo em vista que as crenças das pessoas em sua eficácia pessoal ocupam papel central no processo regulatório da TSG. A teoria social cognitiva fornece um grande corpo de conhecimento particularizado sobre como desenvolver as estruturas cognitivas e alistar os processos do sistema do self que governam a adaptação e mudança humana (BANDURA, 1986, 1997). Ela especifica os mecanismos psicológicos pelos quais as culturas organizacionais podem afetar o comportamento individual (WOOD; BANDURA, 1989).

Para Bandura (1997) quanto maior a autoeficácia de uma pessoa, maior a probabilidade de se envolver e persistir no comportamento relacionado à tarefa. Por outro lado, Powers, (1991) considera que as crenças de autoeficácia podem ser usadas para construir uma percepção do estado atual de uma pessoa.

Gardner e Pierce (1998) relatam que funcionários altamente auto eficazes acreditam que provavelmente terão sucesso na maioria ou em todas as suas obrigações e responsabilidades de trabalho. Nesse ponto de vista, Bandura e Locke (2003) mencionam a autoeficácia percebida é medida em termos de julgamentos de capacidades pessoais e da força dessa crença. De acordo com Markham, Balkin e Baron (2002), a autoeficácia percebida é central para a maioria 
do funcionamento humano e, uma vez que as ações são baseadas mais no que as pessoas acreditam do que no que é objetivamente verdadeiro, os pensamentos são um precursor potente para o nível de motivação, estados afetivos e ações.

De acordo com Bandura (1977a), na maioria dos estudos, as medidas de expectativas referem-se principalmente às esperanças das pessoas por resultados favoráveis, e não ao seu senso de domínio pessoal. Entretanto, para o autor, as expectativas de eficácia variam em diversas dimensões que têm implicações importantes no desempenho, que segundo ele, podem diferir em: Magnitude, a qual aplica-se ao nível de dificuldade da tarefa que uma pessoa acredita que pode alcançar; Generalidade, em que algumas experiências criam expectativas de domínio circunscritas, enquanto outras instilam um senso de eficácia mais generalizado que se estende muito além da situação específica de tratamento e Intensidade, onde expectativas fracas são facilmente extinguíveis por experiências desconfirmadoras, ao passo que indivíduos que possuem fortes expectativas de domínio perseverarão em seus esforços de enfrentamento, apesar das experiências desconfirmadoras.

Segundo Bandura (1997), uma forte crença na eficácia do desempenho de alguém é essencial para mobilizar e sustentar o esforço necessário para ter sucesso. Além disso, as organizações que incorporaram atividades de aprimoramento da autoeficácia em suas práticas de Gestão de Recursos Humanos tendem a desfrutar de maior produtividade, inovação e engajamento, além de diminuir o estresse, desgaste e rotatividade (BANDURA, 2000).

Wood e Bandura (1989) relatam que as crenças das pessoas sobre sua eficácia podem ser acalmadas e fortalecidas através de quatro fontes principais: experiências de domínio, modelagem, persuasão verbal e julgamentos de seus estados fisiológicos. As 'experiências de domínio' são a maneira mais eficaz de os indivíduos desenvolverem um forte senso de eficácia. É especialmente influente porque se baseia em experiências de domínio pessoal. Os sucessos aumentam as expectativas de domínio; falhas repetidas os reduzem, principalmente se os contratempos ocorrerem no início do curso dos eventos. Na 'modelagem', os modelos proficientes constroem autoconfianças de capacidade, transmitindo aos observadores estra- 
tégias eficazes para gerenciar diferentes situações. Ela também afeta as crenças de autoeficácia por meio de um processo de comparação social, onde as pessoas julgam parcialmente suas capacidades em comparação com outras. $\mathrm{Na}$ 'persuasão verbal', os motivadores de sucesso e construtores de eficiência fazem mais do que transmitir avaliações positivas. Além de elevar a crença das pessoas em suas capacidades, eles atribuem tarefas a elas de maneiras que trazem sucesso e evitam colocá-las prematuramente em situações nas quais provavelmente irão falhar. As pessoas também confiam parcialmente em 'julgamentos de seus estados fisiológicos' quando avaliam suas capacidades. Elas interpretam sua excitação emocional e tensão como sinais de vulnerabilidade a um mau desempenho.

A crença das pessoas em sua eficácia também pode afetar seu bem-estar psicológico e desempenho por meio de vários processos intervenientes. As pessoas tendem a evitar atividades e situações que acreditam exceder suas capacidades de enfrentamento, mas prontamente realizam atividades desafiadoras e escolhem ambientes sociais que julgam capazes de administrar. Sendo assim, o desenvolvimento pessoal por meio das competências é definido de acordo com as percepções sobre as influências sociais nos ambientes selecionados (WOOD; BANDURA, 1989).

A partir do presente estudo observou-se que nos últimos 5 anos sobrepõe-se estudos envolvendo a autoeficácia empreendedora, a criativa e a ocupacional. Sendo assim, destaca-se seus principais conceitos. AEE foi definida pelo autor como a capacidade do indivíduo de se envolver e executar com sucesso quaisquer atividades empreendedoras. Boyd e Vozikis (1994) argumentam que a autoeficácia é proposta como uma variável explicativa importante ao determinar a força das intenções empresariais e a probabilidade de que intenções resultarão em ações empreendedoras.

A autoeficácia criativa (AEC) é definida por Tierney e Farmer (2002) como a crença de que alguém tem a capacidade de produzir resultados criativos. Segundo Gong, Huang, Farh (2009), ela é baseada no conhecimento e nas habilidades de uma pessoa que possibilitam a criatividade. Conforme esses autores, a AEC também pode refletir a motivação intrínseca para se engajar em atividades criativas. Como tal, deve ser um precursor poderoso para a criatividade, 
mediando a influência da liderança transformacional e orientação de aprendizagem do funcionário. Além disso, Christensen-Salem et al. (2020) identificaram que a AEC é um fator determinante para saber se os indivíduos irão se aproximar ou evitar uma tarefa, sugerindo que ela pode ser um fator importante que estimula o crescimento, que por sua vez está associado ao desempenho criativo aprimorado.

Outra questão é que ela também fornece aos indivíduos a crença de que eles têm a capacidade de reunir informações relevantes e, portanto, serão mais dispostos a investir recursos cognitivos no desenvolvimento de ideias e soluções exclusivas (Farmer; Tierney, 2017). Por fim, Fuller et al. (2018) estabelecem uma correlação com a autoeficácia empreendedora, ao dizer que, como esta incorpora muitos aspectos relacionados à criatividade relevantes para o domínio empreendedor, a AEC deve promover sentimentos de capacidade empreendedora que leva à autoeficácia empreendedora. Portanto, os autores esperam que a personalidade proativa esteja positivamente relacionada à autoeficácia empreendedora e que essa relação seja mediada pela AEC porque pessoas altamente proativas se sentirão capazes de desempenhar funções amplas.

Quanto à autoeficácia ocupacional (AEO), a teoria social cognitiva de Bandura (1977a) afirma que o ambiente social pode aumentar a AEO de um indivíduo pela entrega de feedback positivo ('persuasão social') e oferecendo oportunidades de aprender com os outros ('experiência vicária'). Para Schyns e Collani (2002), AEO se refere a crença de uma pessoa em sua própria capacidade e competência para desempenhar com sucesso e eficácia em diferentes situações e em diferentes tarefas em um trabalho. Na mesma linha, estão os resultados do estudo de Khalil e Siddiqui (2019), os quais sustentaram que a confiança dos funcionários e a AEO atuam como ferramenta para mediar e fortalecer a relação entre liderança autêntica e engajamento no trabalho.

\section{MÉTodo}

Este estudo foi desenvolvido a partir de pesquisa bibliométrica e os dados foram coletados na Coleção Principal do banco de dados da Web of Science da Clarivate Analytics. A consulta teve como palavra-chave definida self efficacy e a busca foi realizada por meio 
do campo "título", que traz todas as publicações de periódicos, conferências, livros ou capítulos de livro, que contenham tais palavras em seus títulos. Como resultado de tal busca foram apresentadas 14.169 publicações, no intervalo de tempo estipulado entre os anos de 2000 à 2020. Esse resultado foi refinado pelo tipo de documento "artigo", obtendo-se o quantitativo de 10.251 itens.

Para a definição do ano inicial, considerou-se a lista dos 10 artigos mais citados, a qual contém o artigo "Research report: The evolving relationship between general and specific computer self-efficacyAn empirical assessment", publicado no ano 2000. Além disso, a partir deste ano a pesquisa sobre o tema começou a crescer, sendo que a partir de 2010, esse incremento chega próximo a uma exponencial e, portanto, o período de 2010 até 2020 é que será considerado para algumas análises.

Para a definição dos critérios estipulou-se que a pesquisa seria realizada em periódico revisado por pares, por ser uma revisão de um trabalho científico elaborada por especialistas da área do conhecimento do trabalho submetido para avaliação. Os dados descritivos de cada artigo do banco de dados final (ou seja, título, autor, fonte, DOI, ano de publicação, palavras chave/ palavras chave plus e resumo) foram importados para um arquivo do software Microsoft Excel. O principal motivo para usá-lo foi o valor agregado do uso de tabelas dinâmicas para classificar, contar e resumir uma grande quantidade de dados em uma planilha.

Considerando que o objetivo da pesquisa busca analisar o tema na área de gestão, foram selecionadas apenas as categorias de Management e Business (Gestão ou Negócio), já que as demais não satisfaziam o interesse desta pesquisa. Fundamentando-se nessas categorias então, chegou-se a população final da pesquisa, no caso, 519 artigos. Tais publicações foram agrupadas e classificadas, gerando um conjunto de dados que possibilitou uma análise geral desses artigos, assim como uma detalhada dos objetivos, principais resultados e considerações finais/conclusões dos 10 artigos mais citados. Essas informações foram categorizadas conforme as subseções apresentadas na sequência. 


\section{AGRUPANDO, RESUMINDO E RELATANDO OS RESULTADOS}

Este item apresenta os resultados gerais referentes aos 519 artigos analisados, considerando-se o objetivo da pesquisa de analisar o panorama geral das publicações sobre autoeficácia, na área de gestão, a partir de uma revisão bibliométrica.

\section{Áreas de conhecimento e categorias}

A Web of Science atribui pelo menos uma categoria de assunto a todos os periódicos e livros cobertos pela sua coleção principal. Nesse sentido, como resultado da presente pesquisa obteve-se as categorias conforme demonstra o Quadro 1. A análise de tal quadro demonstra o resultado já esperado, considerando que a busca foi pré-refinada por estas categorias, qual seja, Gestão e Negócios. O Quadro 1 apresenta os dez primeiros classificados, por ordem decrescente de contagem de registros, sendo "1" a contagem mínima considerada.

Quadro 1: Quantidade de artigos publicados por Categoria

\begin{tabular}{|l|l|l|}
\hline Categorias & Contagem do registro & \% de 519 \\
\hline Gestão & 388 & 74,76 \\
\hline Negócios & 209 & 40,27 \\
\hline Psicologia aplicada & 156 & 30,06 \\
\hline $\begin{array}{l}\text { Trabalho de Relações In- } \\
\text { dustriais }\end{array}$ & 27 & 5,20 \\
\hline $\begin{array}{l}\text { Ciência da Computação, } \\
\text { Sistemas de Informação }\end{array}$ & 17 & 3,28 \\
\hline $\begin{array}{l}\text { Hospitalidade, Lazer, Es- } \\
\text { porte, Turismo }\end{array}$ & 13 & 2,50 \\
\hline Psicologia Social & 12 & 2,31 \\
\hline $\begin{array}{l}\text { Ciência da informação, } \\
\text { Biblioteconomia }\end{array}$ & 16 & 3,08 \\
\hline Economia & 11 & 2,12 \\
\hline Ética & 9 & 1,73 \\
\hline
\end{tabular}

Fonte: elaborado pela autora com base nos relatórios da Web of Science. 


\section{Autores e Instituições}

O Quadro 2 apresenta os dez autores que mais publicaram sobre o tema, em ordem decrescente, mas o que pode ser percebido pela listagem completa, é que somente um autor publicou seis artigos, dois publicaram cinco e outros quatro publicaram quatro artigos, a maioria do demais autores publicaram apenas um artigo. Os relatórios da Web of Science demonstram que 1291 autores publicaram sobre o tema, sendo que os que mais publicaram, continuam ativos, ou seja, Vancouver JB, com seis artigos, teve sua última publicação em 2019; Schaufeli WB, com cinco artigos, em 2018 e Urban B, também com 5, publicou em 2020. Embora o país que mais publique seja os EUA, apenas Vancouver é americano, Schaufeli e Urban são da Bélgica e da Alemanha, respectivamente.

Quadro 2: Quantidade de artigos publicados por Autor

\begin{tabular}{|l|l|l|}
\hline Autores & Contagem do Registro & \% de 519 \\
\hline Vancouver JB & 6 & 1,16 \\
\hline Schaufeli WB & 5 & 0,96 \\
\hline Urban B & 5 & 0,96 \\
\hline Borgogni L & 4 & 0,77 \\
\hline Schmidt AM & 4 & 0,77 \\
\hline Thatcher JM & 4 & 0,77 \\
\hline Walumbwa FO & 4 & 0,77 \\
\hline Alalwan AA & 3 & 0,58 \\
\hline Azeem MU & 3 & 0,58 \\
\hline Baron RA & 3 & 0,58 \\
\hline
\end{tabular}

Fonte: elaborado pela autora com base nos relatórios da Web of Science.

Um fato notável entre esses autores é que Schaufeli e Urban seguem Bandura, tentando confirmar seus estudos, enquanto as pesquisas de Vancouver questionam, buscando encontrar efeitos negativos da autoeficácia. O Quadro 3 exibe as 10 primeiras Instituições-chave às quais os autores foram associados, destacando-se as americanas. 
Quadro 3: Quantidade de artigos publicados por Instituição

\begin{tabular}{|l|l|l|}
\hline Instituição & Contagem do Registro & \% de 519 \\
\hline Michigan State University & 13 & 2,50 \\
\hline University Minnesota & 10 & 1,93 \\
\hline $\begin{array}{l}\text { Georgia Institute of Tech- } \\
\text { nology }\end{array}$ & 9 & 1,73 \\
\hline Monash University & 9 & 1,73 \\
\hline University of Amsterdam & 7 & 1,35 \\
\hline $\begin{array}{l}\text { Eindhoven University Te- } \\
\text { chnology }\end{array}$ & 6 & 1,16 \\
\hline $\begin{array}{l}\text { Hong Kong Polytechnic } \\
\text { University }\end{array}$ & 6 & 1,16 \\
\hline Ohio University & 6 & 1,16 \\
\hline Portland State University & 6 & 1,16 \\
\hline University of Illinois & 6 & 1,16 \\
\hline
\end{tabular}

Fonte: elaborado pela autora com base nos relatórios da Web of Science.

\section{Título das Fontes}

A pesquisa empírica sobre autoeficácia está espalhada por uma ampla gama de periódicos (161 periódicos), indicando que não há, por parte dos autores, um periódico de preferência para o tema. Este fato pode estar relacionado a características genéricas do tema em questão bem como a sua muldisciplinariedade. A maioria dos periódicos publicaram apenas um artigo, mas destaca-se o Journal of Applied Psychology com 737 artigos publicados, condição que possibilita ser considerado como a referência do tema. Trata-se de um periódico acadêmico mensal, publicado pela American Psychological Association, a qual enfatiza a publicação de investigações originais que contribuem com novos conhecimentos e entendimentos para os campos da psicologia (APA, 2021).

O periódico Personnel Review publica artigos de uma variedade de tradições teóricas e metodológicas e o Career Development International fornece uma plataforma de pesquisa nas áreas de Carreiras e Desenvolvimento que atua com questões de teorias e desenvolvimento de teoria, bem como com estratégia de carreira organizacional, política e prática. 
A fonte do primeiro artigo mais citado - Organizational Research Methods - não consta na lista das 10 primeiras fontes, nem mesmo na lista integral que apresenta os resultados de publicações de até 5 artigos. Este periódico retrata desenvolvimentos metodológicos relevantes para pesquisadores que trabalham em ciências organizacionais. O segundo, o terceiro e o nono artigos mais citados foram publicados no Journal of Applied Psychology, primeira fonte classificada. O quarto e o quinto não aparecem na lista de fontes, foram publicados no The Academy of Management Journal. Já o sexto e oitavo mais citados constam na sétima fonte, enquanto que o sétimo e o décimo artigos mais citados, também não constam nas fontes tendo sido publicados no Information Systems Research e MIS Quarterly respectivamente.

Quadro 4: Quantidade de artigos publicados por fonte

\begin{tabular}{|l|l|l|}
\hline Fontes & $\begin{array}{l}\text { Contagem do } \\
\text { Registro }\end{array}$ & $\begin{array}{l}\% \\
\text { de 519 }\end{array}$ \\
\hline Journal of Applied Psychology & 37 & 7,13 \\
\hline Personnel Review & 14 & 2,70 \\
\hline Career Development International & 13 & 2,50 \\
\hline $\begin{array}{l}\text { European Journal of Work And Organi- } \\
\text { zational Psychology }\end{array}$ & 11 & 2,12 \\
\hline Journal of Organizational Behavior & 11 & 2,12 \\
\hline $\begin{array}{l}\text { Organizational Behavior and Human } \\
\text { Decision Processes }\end{array}$ & 10 & 1,93 \\
\hline Entrepreneurship Theory and Practice & 9 & 1,73 \\
\hline Journal of Business Ethics & 9 & 1,73 \\
\hline Human Relations & 8 & 1,54 \\
\hline $\begin{array}{l}\text { International Journal of Entrepreneurial } \\
\text { Behavior Research }\end{array}$ & 8 & 1,54 \\
\hline
\end{tabular}

Fonte: elaborado pela autora com base nos relatórios da Web of Science 


\section{Ano das publicações}

A Figura 2 apresenta o número de documentos publicados durante o ano, de acordo com a pesquisa no banco de dados WOS, no período de 2000 a 2020. A figura mostra que a partir do ano 2000, houve um aumento significativo na pesquisa sobre o tema, sendo que a partir de 2010, esse incremento pode ser considerado próximo de uma exponencial. $\mathrm{O}$ ano de 2019 é o que apresenta maior número de publicações, ou seja, 97, valor próximo do dobro que o ano anterior. Salienta-se que, embora a curva apresente um declínio em 2020, isso não significa que o tema vem sendo menos pesquisado, deve-se considerar que a presente pesquisa foi realizada no decorrer do ano de 2020 e que os artigos levam tempo para serem aprovados assim como para serem incluídos nas bases de dados.

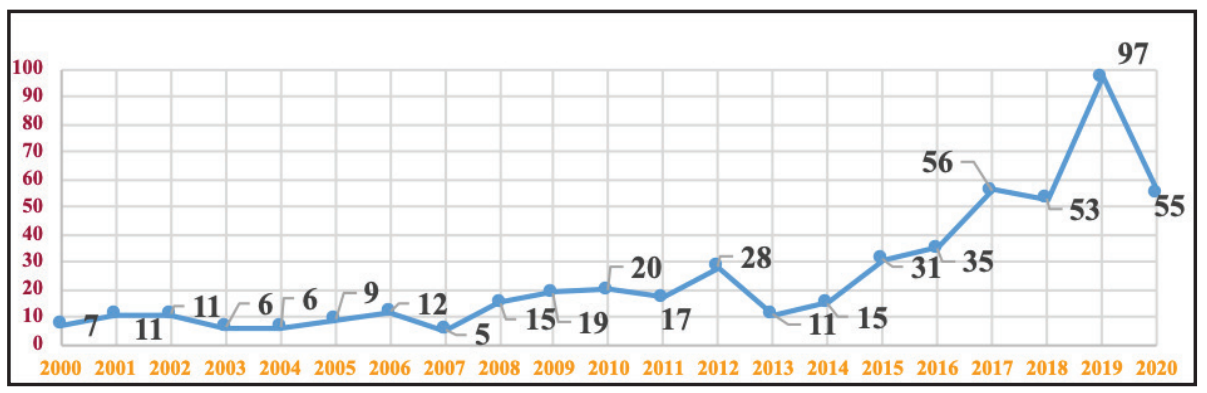

Figura 2- Quantidade de artigos publicados por ano

Fonte: elaborado pela autora com base nos relatórios da Web of Science

\section{Distribuição geográfica das publicações e idiomas}

A pesquisa empírica referente a autoeficácia foi publicada em 42 países diferentes. Observando a representação do país com base na afiliação de todos os autores listados em cada publicação, os Estados Unidos lideram o ranking de artigos publicados (203), seguidos pela China, Austrália, Taiwan e Canadá e Inglaterra. Tal resultado pode ser explicado devido a literatura em gestão e negócios ser tradicionalmente americana e ao fato que o principal autor do tema 'Albert Bandura', embora Canadense, seja professor da Universidade de Stanford. Em relação ao idioma mais utilizado nos artigos analisados, destaca-se o inglês, com 513 trabalhos publicados, representando 98,84\% da amostra. O Quadro 6 apresenta os dez países que mais publicaram. 
Quadro 6: Quantidade de artigos publicados por país

\begin{tabular}{|l|l|l|}
\hline Países & Contagem do Registro & \% de 519 \\
\hline EUA & 203 & 39,11 \\
\hline China & 50 & 9,63 \\
\hline Austrália & 37 & 7,13 \\
\hline Taiwan & 34 & 6,55 \\
\hline Canadá & 30 & 5,78 \\
\hline Inglaterra & 30 & 5,78 \\
\hline Países Baixos & 25 & 4,82 \\
\hline Índia & 22 & 4,24 \\
\hline Paquistão & 20 & 3,85 \\
\hline Alemanha & 19 & 3,66 \\
\hline
\end{tabular}

Fonte: elaborado pela autora com base nos relatórios da Web of Science

\section{Publicações mais citadas}

O Quadro 8 apresenta a quantidade, ano de publicação e a média anual de citações dos dez artigos mais citados em pesquisas sobre Autoeficácia, organizado por ordem decrescente de contagem de registros. Destaca-se que os autores mais citados, não são os que mais publicam, estes sequer aparecem entre os dez primeiros em número de citações. Vancouver JB, o autor que mais publicou (6 artigos) aparece na $13^{\circ}, 14^{\circ}$ e 17 posições da lista de mais citados, Schaufeli WB (5 artigos), aparece na $66^{\circ}$ e $123^{\circ}$ posições, enquanto que Urban B (5 artigos) sequer é mencionado.

Os principais autores dos três primeiros artigos são americanos, condizendo com o país que mais publica. Destaca-se a expressividade do artigo mais citado, Validation of a new general self-efficacy scale, o qual obteve 1000 citações. Nesse artigo os autores desenvolveram uma nova escala geral de autoeficácia e compararam suas propriedades psicométricas e validade com a Escala Geral de Autoeficácia de Sherer et al. (1982). O segundo autor mais citado, Bandura A. também não aparece entre os que mais publicam, o que se explica pela maioria de suas publicações serem anteriores ao pe- 
ríodo de tempo deste estudo. Destaca-se que Bandura A. é o autor de referência no assunto, o que pode ser observado pelos trabalhos relacionados nas referências deste estudo. Além disso, exalta-se tal autor por ser considerado um dos dez psicólogos vivos mais citados do mundo, tendo obtido 927 citações, em sua mais recente publicação: Negative self-efficacy and goal effects revisited, publicada em 2003, quando o autor possuía 78 anos.

Quadro 7: Publicações mais citadas por ano (continua)

\begin{tabular}{|c|c|c|c|c|c|}
\hline Título & Autor & Fonte & Ano & Total & Média \\
\hline $\begin{array}{l}\text { 1) Validation of a new general } \\
\text { self-efficacy scale }\end{array}$ & $\begin{array}{l}\text { Chen, G; Gully, } \\
\text { SM; Eden, D }\end{array}$ & $\begin{array}{l}\text { Organi- } \\
\text { zational } \\
\text { Research } \\
\text { Methods }\end{array}$ & 2001 & 1000 & 50 \\
\hline $\begin{array}{l}\text { 2) Negative self-efficacy and } \\
\text { goal effects revisited }\end{array}$ & $\begin{array}{l}\text { Bandura, A; } \\
\text { Locke, EA }\end{array}$ & $\begin{array}{l}\text { Journal of } \\
\text { Applied } \\
\text { Psychology }\end{array}$ & 2003 & 927 & 51,5 \\
\hline $\begin{array}{l}\text { 3) The mediating role of self- } \\
\text { efficacy in the development of } \\
\text { entrepreneurial intentions }\end{array}$ & $\begin{array}{l}\text { Zhao, H; Sei- } \\
\text { bert, SE; Hills, } \\
\text { GE }\end{array}$ & $\begin{array}{l}\text { Journal of } \\
\text { Applied } \\
\text { Psychology }\end{array}$ & 2005 & 892 & 55,75 \\
\hline $\begin{array}{l}\text { 4) Creative self-efficacy: } \\
\text { Its potential antecedents } \\
\text { and relationship to creative } \\
\text { performance }\end{array}$ & $\begin{array}{l}\text { Tierney, P; } \\
\text { Farmer, SM }\end{array}$ & $\begin{array}{l}\text { Academy } \\
\text { of Mana- } \\
\text { gement } \\
\text { Journal }\end{array}$ & 2002 & 842 & 44,32 \\
\hline $\begin{array}{l}\text { 5) Employee Learning } \\
\text { Orientation, Transformatio- } \\
\text { nal Leadership, and Employee } \\
\text { Creativity: The Mediating } \\
\text { Role of Employee Creative } \\
\text { Self-Efficacy }\end{array}$ & $\begin{array}{l}\text { Gong, Yaping; } \\
\text { Huang, Jia-Chi; } \\
\text { Farh, Jiing-Lih }\end{array}$ & $\begin{array}{l}\text { Academy } \\
\text { of Mana- } \\
\text { gement } \\
\text { Journal }\end{array}$ & 2009 & 673 & 56,08 \\
\hline $\begin{array}{l}\text { 6) Gender, entrepreneurial } \\
\text { self-efficacy, and entrepre- } \\
\text { neurial career intentions: } \\
\text { Implications for entrepreneur- } \\
\text { ship education }\end{array}$ & $\begin{array}{l}\text { Wilson, Fiona; } \\
\text { Kickul, Jill; Mar- } \\
\text { lino, Deborah }\end{array}$ & $\begin{array}{l}\text { Entrepre- } \\
\text { neurship } \\
\text { Theory and } \\
\text { Practice }\end{array}$ & 2007 & 622 & 44,43 \\
\hline $\begin{array}{l}\text { 7) Research report: The } \\
\text { evolving relationship between } \\
\text { general and specific computer } \\
\text { self-efficacy - An empirical } \\
\text { assessment }\end{array}$ & $\begin{array}{l}\text { Agarwal, R; } \\
\text { Sambamurthy, } \\
\text { V; Stair, RM }\end{array}$ & $\begin{array}{l}\text { Information } \\
\text { Systems } \\
\text { Research }\end{array}$ & 2000 & 374 & 17,81 \\
\hline
\end{tabular}


(conclusão)

\begin{tabular}{|l|l|l|l|l|c|}
\hline Título & Autor & Fonte & Ano & Total & Média \\
\hline $\begin{array}{l}\text { 8) Entrepreneurial } \\
\text { Self-Efficacy: Refining } \\
\text { the Measure }\end{array}$ & $\begin{array}{l}\text { McGee, Jeffrey E.; } \\
\text { Peterson, Mark; } \\
\text { Mueller, Stephen L.; } \\
\text { Sequeira, Jennifer M. }\end{array}$ & $\begin{array}{l}\text { Entrepreneu- } \\
\text { rship Theory } \\
\text { and Practice }\end{array}$ & 2009 & 371 & 30,92 \\
\hline $\begin{array}{l}\text { 9) The role of different } \\
\text { levels of leadership in } \\
\text { predicting self- and } \\
\text { collective efficacy: } \\
\begin{array}{l}\text { Evidence for discon- } \\
\text { tinuity }\end{array}\end{array}$ & Chen, GL; Bliese, PD & $\begin{array}{l}\text { Journal of } \\
\text { Applied } \\
\text { Psychology }\end{array}$ & 2002 & 332 & 17,47 \\
\hline $\begin{array}{l}\text { 10) An empirical } \\
\text { examination of indi- } \\
\text { vidual traits as ante- } \\
\text { cedents to computer } \\
\text { anxiety and computer } \\
\text { self-efficacy }\end{array}$ & $\begin{array}{l}\text { Thatcher, JB; Per- } \\
\text { rewé, PL }\end{array}$ & $\begin{array}{l}\text { Journal of } \\
\text { Applied } \\
\text { Psychology }\end{array}$ & 2002 & 316 & 16,63 \\
\hline
\end{tabular}

Fonte: elaborado pela autora com base nos relatórios da Web of Science

\section{Análise detalhada dos artigos mais citados}

Este item apresenta uma análise detalhada dos objetivos, principais resultados e considerações finais/conclusões dos 10 artigos mais citados, tendo em vista o limite de espaço. A partir da análise observa-se que o principal objetivo dos artigos é investigar o desempenho dos funcionários e identificar quais são os fatores que podem influenciá-los, o que é uma das prioridades mais importantes no gerenciamento de recursos humanos.

No primeiro artigo mais citado, Validation of a New General Self-Efficacy Scale, os autores desenvolveram uma nova escala de autoeficácia (NGSE) e compararam suas propriedades psicométricas e validade com a Escala Geral de Autoeficácia (SGSE) (SHERER, et al., 1982). O estudo descobriu que a NGSE tem maior validade de construto do que a escala SGSE, além de demonstrar alta confiabilidade, embora menor que a SGSE, também previu autoeficácia específica (SSE) para uma variedade de tarefas em vários contextos 
e moderou a influência do desempenho anterior na formação de SSE subsequente (CHEN; GULLY; EDEN; 2001).

No segundo artigo mais citado, Negative Self-Efficacy and Goal Effects Revisited, os autores abordam a verificação das propriedades funcionais das crenças de autoeficácia e documentam como as crenças de autoeficácia operam em conjunto com os sistemas de metas dentro de uma teoria sociocognitiva de autorregulação em contraste com o foco da teoria de controle na redução da discrepância. Os resultados contradizem outros estudos, os quais indicaram que a crença nas próprias capacidades e objetivos pessoais é autodebilitante (BANDURA; LOCKE, 2003).

O terceiro artigo, The Mediating Role of Self-Efficacy in the Development of entrepreneurial intentions, teve como objetivo investigar o papel mediador da autoeficácia no desenvolvimento das intenções dos alunos de se tornarem empreendedores. Os resultados mostraram que os efeitos da aprendizagem percebida em cursos relacionados ao empreendedorismo, experiência empreendedora anterior e propensão ao risco sobre as intenções empreendedoras foram totalmente mediados pela autoeficácia empreendedora (ZHAO, SEIBERT; HILLS 2005).

Tierney e Farmer (2002) testaram a autoeficácia criativa no quarto artigo, Creative self-efficacy: Its Potential Antecedents and Relationship to Creative Performance, explorando as crenças dos funcionários de que eles podem ser criativos em suas funções de trabalho. Os resultados apoiam a validade discriminante do construto e indicam que a estabilidade no trabalho, a autoeficácia no trabalho, o comportamento do supervisor e a complexidade do trabalho contribuem para as crenças de eficácia criativa.

No quinto artigo, Employee Learning Orientation, Transformational Leadership, and Employee Creativity: The Mediating Role of Employee Creative Self-Efficacy, Gong, Huang, Farh (2009) examinaram a relação entre a criatividade do funcionário e o desempenho no trabalho, além de identificar duas variáveis pessoais e situacionais relacionadas à aprendizagem examinando seus efeitos na criatividade do funcionário por meio de sua autoeficácia criativa. Descobriram que a criatividade dos funcionários estava positivamente relacionada às vendas dos funcionários e ao desempenho do funcionário ava- 
liado pelo supervisor, as variáveis também estavam positivamente relacionadas à criatividade do funcionário, e essas relações eram mediadas pela sua autoeficácia criativa.

Wilson, Kickul e Marlino (2007), examinaram no sexto artigo Gender, Entrepreneurial Self-Efficacy, and Entrepreneurial Career Intentions: Implications for Entrepreneurship Education, as relações entre gênero, autoeficácia empreendedora e intenções empreendedoras em adolescentes e alunos adultos de mestrado em administração de empresas (MBA). Encontraram fortes efeitos de gênero na autoeficácia empresarial e nas intenções no nível do ensino fundamental/ médio e embora não tenham medido os estereótipos de gênero para diferentes planos de carreira, seus resultados sugerem que o empreendedorismo ainda pode ser percebido como um campo "masculino" e que as mulheres jovens podem estar limitando suas aspirações de carreira porque sentem que não têm as aptidões necessárias. Segundo eles, a educação para o empreendedorismo pode ser posicionada como um equalizador, possivelmente reduzindo os efeitos limitantes da baixa autoeficácia e, em última análise, aumentando as chances de criação de empreendimentos bem-sucedidos por mulheres.

O sétimo artigo, o único através de estudo longitudinal, amplia o entendimento atual sobre o conceito de autoeficácia no contexto de software de computador. Os autores descrevem como dois tipos amplos de crenças de autoeficácia do computador (CSE), autoeficácia geral e autoeficácia específica da tarefa, são construídas em diferentes tarefas de computação, sugerindo que as crenças de CSE gerais iniciais irão predizer fortemente as crenças de CSE específicas subsequentes. Os resultados também mostram que os julgamentos de autoeficácia servem como antecedentes-chave do esforço cognitivo percebido (facilidade de uso) associado ao uso da tecnologia. Outra postulação confirmada foi que os julgamentos de autoeficácia no domínio da tarefa de computação são fortemente influenciados pela extensão em que os indivíduos acreditam que são pessoalmente inovadores no que diz respeito à tecnologia da informação (AGARWAL; SAMBAMURTHY; STAIR, 2000).

McGee et al. (2009), no oitavo artigo, dentro de uma estrutura de processo de criação de novos empreendimentos, desenvolvem e testam um instrumento de autoeficácia empreendedora (ESE) mul- 
tidimensional, em uma amostra diversa que inclui empreendedores nascentes, além de discutir implicações para a teoria do empreendedorismo e educação para o empreendedorismo. Os resultados do estudo sugeriram que o ESE é melhor visto como uma construção multidimensional e ademais esta medida de ESE refinada parece particularmente apropriada para examinar o comportamento de empreendedores nascentes. Os autores ainda encontraram uma relação positiva entre o empreendedorismo nascente e as cinco dimensões da ESE. Para eles, esses resultados fornecem uma compreensão mais estruturada do ESE e sua capacidade de avaliar o aumento da confiança de empreendedores nascentes nas dimensões de uma estrutura de processo de criação de novos empreendimentos.

O nono artigo identificou descontinuidades potenciais nos antecedentes das crenças de eficácia entre os níveis de análise, com um foco particular no papel do clima de liderança em diferentes níveis organizacionais. As análises em nível de grupo identificaram o clima de liderança em um nível organizacional superior como o mais forte preditor de eficácia coletiva. Especificamente, a experiência de trabalho, a clareza do papel e a tensão psicológica foram considerados preditores diretos importantes de autoeficácia, enquanto o clima de liderança foi considerado apenas um indicador distal. Em contraste, o clima de liderança (particularmente o clima de liderança de nível superior) foi considerado um preditor imediato da eficácia coletiva, mesmo enquanto controlava os níveis médios de experiência de trabalho, clareza de papéis e tensão psicológica em grupos. Descobertas são importantes porque supõe como infundadas o que os pesquisadores assumiram amplamente até o momento, ou seja, que os modelos de eficácia pessoal e coletiva são homólogos. Assim, as intervenções direcionadas ao aumento da autoeficácia não necessariamente aumentariam a eficácia coletiva e vice-versa (CHEN; BLIESE, 2002).

O décimo e último artigo, articulou e testou um modelo conceitual que postulou três características estáveis (ou seja, Inovação Pessoal na Tecnologia da Informação (PIIT), Afetividade Negativa (NA) e Ansiedade Traço (TA) que influenciaria duas diferenças individuais dinâmicas (ou seja, ansiedade do computador (CA) e Autoeficácia do Computador (CSE)). Conforme os autores, embora 
não tenha havido efeitos para NA, o suporte para as relações entre PIIT e TA com CA foi suportado. Dada a influência de CA e CSE nas crenças de computação, os resultados ressaltam a importância de estender a rede nomológica em torno das diferenças individuais dinâmicas no contexto de TI. Do ponto de vista teórico, os resultados ajudam a aprofundar a compreensão da rede nomológica entre as diferenças individuais que levam à autoeficácia do computador, enquanto que de uma perspectiva prática, as descobertas podem ajudar os gerentes de TI a projetar programas de treinamento que aumentem, de forma mais eficaz, a autoeficácia do computador de usuários com diferentes características de disposição (THATCHER; PERREWÉ, 2002).

\section{CONSIDERAÇões FINAIS}

O presente estudo adotou uma rigorosa análise bibliométrica referente as características da literatura empírica sobre autoeficácia, na área de gestão, com o objetivo de fornecer uma imagem clara e abrangente da pesquisa realizada até o momento.

Houve um interesse crescente na pesquisa empírica sobre o tema ao longo dos últimos anos, o ano de 2019 apresentou o maior número de publicações, resultado que influenciou no incremento de pesquisas da última década, atingindo um acréscimo de $250 \%$, se comparado com a década anterior.

Os estudos relacionados ao tema têm se diversificado nos últimos vinte anos, a maioria delas restringem-se a crenças específicas de variados tópicos, como: autoeficácia do computador, financeira, da amplitude de papéis, entre diversas outras. Embora persista a diversidade, nos últimos 5 anos, sobrepõe-se estudos envolvendo a autoeficácia empreendedora, a criativa e a ocupacional. Apesar da diversidade nos assuntos, observa-se uma escassez de estudos longitudinais, como examinar quem realmente se torna ou tem sucesso como empreendedor (ZHAO; SEIBERT; HILLS, 2005), ou para avaliar a influência de traços amplos e específicos da situação nas ligações entre a autoeficácia do computador e a ansiedade ao longo do tempo (THATCHER; PERREWÉ, 2002).

Essa diversidade também se aplica ao número de autores, considerando que mais de 1200 contribuíram para a pesquisa empírica 
sobre tema, ademais os autores que mais publicaram, continuam ativos, sendo eles Vancouver JB, Schaufeli WB e Urban B. Um fato notável entre esses autores é que Schaufeli e Urban seguem Bandura, tentando confirmar seus estudos, enquanto que as pesquisas de Vancouver questionam, buscando sempre encontrar efeitos negativos da autoeficácia.

O país que mais publica é os EUA, entretanto, entre os autores, apenas Vancouver é americano, Schaufeli e Urban são da Bélgica e da Alemanha, respectivamente. Entre as Instituições-chave, também se destacando as americanas que são: Michigan State University (EUA), a University Minnesota (EUA), o Georgia Institute of Technology (EUA), e a Monash University (Austrália).

Comparando-se as publicações mais citadas com os autores mais publicados no período pesquisado, constatou-se que os autores mais citados, não são os que mais publicam, estes sequer aparecem entre os dez primeiros em número de citações. Além disso, deve-se enfatizar que as publicações sobre autoeficácia buscam investigar o desempenho dos funcionários e identificar os fatores que o influenciam, uma das prioridades mais importantes no gerenciamento de recursos humanos.

Os estudos sobre o tema demonstram como pessoas autoeficazes são melhores funcionários no sentido que desempenham melhor suas funções, pois são mais eficientes, gostam de desafios, sabem agir em situações de pressão, são mais criativas e ou empreendedoras, conforme o ambiente exige, já que se adaptam a diversas situações, dentre outras diversas características que as tornam diferenciadas. Entretanto, estudos futuros precisam confirmar de forma prática como se localizam estas pessoas, quais as formas, se é que elas existem, de promover a autoeficácia? Os estudos já realizados também devem ser testados em formato longitudinal, com o intuito de demonstrar quais fatores podem afetar ou influenciar este comportamento. Igualmente devem ser analisados se as características de gêneros são fatores influenciadores da autoeficácia geral, visto que os estudos que fizeram esta análise referem-se a autoeficácia específica. Também são necessários estudos que examinem a relação entre a autoeficácia e outros construtos e se ela medeia essas relações.

Finalmente, quanto às limitações do estudo, aponta-se a uti- 
lização de apenas uma base de dados específica além de ter sido feita apenas uma análise bibliométrica. Sendo assim, sugere-se a realização de estudos futuros em outras bases e ou uma revisão sistemática dos 519 artigos relacionados.

\section{REFERÊNCIAS}

AGARWAL, R.; SAMBAMURTHY, V.; STAIR, R. M. The Evolving Relationship Between General and Specific Computer Self-Efficacy - An Empirical Assessment. Information Systems Research, Vol. 11, N. 4, pp. 418-430, 2000.

AMERICAN PSYCHOLOGICAL ASSOCIATION (APA). Washington, 2021. Disponível em: https://www.apa.org/pubs/journals, 2021.

BANDURA, A.; LOCKE, E. A. Negative Self-Efficacy and Goal Effects Revisited. Journal of Applied Psychology, Vol. 88, No. 1, 87-99, 2003

BANDURA, A. Cultivate self-efficacy for personal and organizational effectiveness, in Locke, A. (Ed.), Handbook of Principles of Organization Behavior, Blackwell, Oxford, pp. 120-136, 2000.

BANDURA, A. A social cognitive theory of personality. In: L. Pervin \& O. John (Ed.), Handbook of personality (2nd ed., pp. 154-196). New York: Guilford Publications. (Reprinted in D. Cervone \& Y. Shoda [Eds.], The coherence of personality. New York: Guilford Press., 1999 BANDURA, A. Self-efficacy: The exercise of control. New York: Freeman, 1997.

BANDURA, A.; WOOD, R. Effect of perceived controllability and performance standards on self-regulation of complex decision making. Journal of Personality and Social Psychology, 56, 805-814, 1989

BANDURA, A. Social foundations of thought and action: A social cognitive theory. Englewood Cliffs, NJ: Prentice-Hall, 1986.

BANDURA, A. 1982 Self-efficacy mechanism in human agency. American Psychologist, 37, $122-147,1982$

BANDURA, A. The self system in reciprocai determinism. American Psychologist, v. 33, p. 344-358, 1978.

BANDURA, A.: Self-efficacy: toward a unifying theory of behavioral change. Psychological Review, 84, 191-215., 1977a. http://dx.doi.org/10.1037/0033-295X.84.2.191.

BANDURA, A. Social learning theory. Englewood Cliffs, NJ: Prentice-Hall, 1977b.

BOYD, N.G., VOZIKIS, G.S. The influence of self-efficacy on the development of entrepreneurial intentions and actions. Entrepreneurship Theory and Practice, v 18, p. 63-90, 1994.

CHEN, G.; BLIESE, P. D. The role of different levels of leadership in predicting self- and collective efficacy: evidence for discontinuity. Journal of Applied Psychology, Vol. 87, N. 3, 549-556, 2002. 
CHEN, G.; GULLY, S. M.; EDEN, D. Validation of a New General Self-Efficacy Scale. Organizational CHEN, C. C. C., GREENE, P. G.; CRICK Research Methods, v. 4, n. 1, p. 62-83, 2001. CHRISTENSEN-SALEM, A.; WALUMBWA, F. O.; HSU, C. I-C.; MISATI, E. BABALOLA, M. T.; KIM, K.: Unmasking the creative selfefficacy-creative performance relationship: the roles of thriving at work, perceived work significance, and task interdependence. The International Journal of Human Resource Management, 1466-4399, 2020.

FARMER, S. M.; TIERNEY, P.: Considering creative self-efficacy: Its current state and ideas for future inquiry. Elsevier Inc. doi.org/10.1016/B978-0-12-809790-8.00002-9, 2017.

FULLER, B.; LIU, Y.; BAJABA, S.; MARLER, L. E.; PRATT, J.: Examining how the personality, self-efficacy, and anticipatory cognitions of potential entrepreneurs shape their entrepreneurial intentions. Personality and Individual Differences, v 125, p. 120-125, 2018.

GARDNER, D. G.; PIERCE, J. L. Self-esteem and self-efficacy within the organizational context. Group and Organization Management, v. 23, p. 48-70, 1998.

GONG, Y.; HUANG, J-C.; FARH, J-L. Employee Learning Orientation, Transformational Leadership, and Employee Creativity: The Mediating Role of Employee Creative Self-Efficacy. Academy of Management Journal. v. 52, n. 4, 765-778, 2009.

GRETHER, T.; SOWISLO, J. F.; WIESE, B. S. Top-down or bottom-up? Prospective relations between general and domain-specific self-efficacy beliefs during a work-family transition. Personality and Individual Differences, v. 121, p. 131-139, Sep 2017 http://dx.doi.org/10.1016/j. paid.2017.09.021.

KHALIL, S. A.; SIDDIQUI, D. A.: Authentic leadership and work engagement: the mediatory role of employees' trust and occupational self-efficacy. International Journal of Organizational Leadership, v. 8, p. 17-42, 2019.

MARKHAM, G.; BALKIN, D.; BARON, R. Inventors and new venture formation: the effects of general self-efficacy and regretful thinking. Entrepreneurship Theory and Practice, v. 27, n. 2, p. 149-165, 2002.

McGEE, J. E., PETERSON, M.; MUELLER, S. L.; SEQUEIRA, J. M. Entrepreneurialself-efficacy: refining the measure. Entrepreneurship Theory and Practice, 1042-2587, 2009.

POWERS, W. T. Commentary on Bandura's human agency. American Psychologist, 46, 151-153, 1991.

SCHERBAUM, C. A.; COHEN-CHARASH; Y.; KERN, M. J. Measuring general self-efficacy: a comparison of three measures using item response theory. Educational and Psychological Measurement, v. 66, p. 1047-1063, 2006. http://dx.doi.org/10.1177/0013164406288171.

SCHYNS, B.; COLLANI, G. V. A new occupational self-efficacy scale and its relation to personality constructs and organizational variables. European Journal of Work and Organizational Psychology, v. 11, n. 2, p. 219-241, 2002 DOI:10.1080/13594320244000148

SHERER, M.; MADDUX, J. E.; MERCANDANTE, B.; PRENTICE-DUNN, S.; JACOBS, B.; ROGERS, R. W. The self-efficacy scale: construction and validation. Psychological Reports, v. 51, p. 663-671, 1982. 
SCHWARZER, R.; JERUSALEM, M. Generalized self-efficacy scale. In: J. Weinman, S. Wright, $\&$ M. Johnston (Eds.). Measures in health psychology: A user's portfolio. Causal and control beliefs, p. 35-37, 1995 NFER-NELSON: Windsor, UK.

THATCHER, J. B.; PERREWÉ, P. L. An empirical examination of individual traits as antecedents to computer anxiety and computer self-efficacy. MIS Quarterly, Vol. 26, N. 4, pp. 381-396, 2002.

TIERNEY, P; FARMER, S. M. Creative self-efficacy: its potential antecedents and relationship to creative performance. The Academy of Management Journal, Vol. 45, No. 6, pp. 1137114, 2002.

VANCOUVER, J. B.; THOMPSON, C. M.; WILLIAMS, A. A. The changing signs in the relationships between self-efficacy, personal goals and performance. Journal of Applied Psychology, 86, 605-620, 2001

VANCOUVER, J. B.; THOMPSON, C. M. E.; TISCHNER, C.; PUTKA, D. J. Two studies examining the negative effect of self-efficacy on performance. Journal of Applied Psychology, v. 87, n. 3, p. 506-516, 2002.

WILSON, F.; KICKUL, J. MARLINO, D. Gender, entrepreneurial self-efficacy, and entrepreneurial career intentions: implications for entrepreneurship education. Entrepreneurship Theory and Practice, 2007.

WOOD, R.; BANDURA, A. Impact of conceptions of ability on self-regulatory mechanisms and complex decision making. Journal of Personality and Social Psychology, 56, 407-415, 1989.

ZHAO, H. SEIBERT, S. E.; HILLS, G. E. The mediating role of self-efficacy in the development of entrepreneurial intentions. Journal of Applied Psychology, v. 90, n. 6, p. 1265-1272, 2005.

Recebido em: 5-10-2021

Aprovado em: 3-12-2021

Avaliado pelo sistema double blind review.

Disponível em http://mjs.metodista.br/index.php/roc 